\title{
LARGE TRANSCENDENCE DEGREE
}

\author{
ROBERT TUBBS
}

(Received 14 November 1989)

Communicated by J. H. Loxton

\begin{abstract}
In this paper we study the transcendence degree of fields generated over $Q$ by the numbers associated with values of one-parameter subgroups of commutative algebraic groups. We show that in many instances these fields have a large transcendence degree when measured in terms of the available data.

Our method deals with points which are "well distributed" (in a sense which is made precise) among certain algebraic subgroups of the algebraic group under consideration. We verify that these results apply in many classical situations.
\end{abstract}

1991 Mathematics subject classification (Amer. Math. Soc.) $11 \mathrm{~J} 99$.

The purpose of this paper is to study fields of large transcendence degree, over $\overline{\mathbb{Q}}$, which are generated by numbers associated with one-parameter subgroups of commutative algebraic groups. This is not the first such investigation. Our goal here is to both generalize and improve several results which have appeared in recent years. Specifically we apply some ideas from the work of G. Diaz [6] and some of the present author [15], [16] to study algebraic independence in this general setting, which was introduced by $M$. Waldschmidt in [17].

We begin with a description of the basic objects in our study. Let $K$ be an arbitrary subfield of $\mathbb{C}$ and suppose that $G$ is a commutative algebraic group defined over $K$ of dimension $d$. Moreover, letting $\mathbb{G}_{a}$ denote the additive group of complex numbers and $\mathbb{G}_{m}$ the multiplicative group of complex

(C) 1991 Australian Mathematical Society 0263-6115/91 \$A2.00+0.00 
numbers, we assume that $G$ has a decomposition

$$
G=\mathbb{G}_{a}^{d_{0}} \times \mathbb{G}_{m}^{d_{1}} \times G_{2},
$$

with $G_{2}$ a commutative algebraic group, necessarily defined over $K$, of dimension $d_{2}$.

Let $\phi: \mathbb{C} \rightarrow G(\mathbb{C})$ be an analytic homomorphism whose image is Zariski dense in $G(\mathbb{C})$. We assume that $y_{1}, \ldots, y_{l}$ are $\mathbb{Q}$-linearly independent complex numbers with $\phi\left(y_{i}\right) \in G(K)$ for $i=1, \ldots, l$.

Our goal is to provide a lower bound on the transcendence degree of $K$ which is given in terms of $d_{0}, d_{1}, d_{2}$ and $l$. To accomplish this we must make some assumptions on the points $y_{1}, \ldots, y_{l}$ and on the distribution of the points $\phi\left(s_{1} y_{1}+\cdots+s_{l} y_{l}\right), s_{i} \in \mathbb{Z}$, among certain algebraic subgroups of $G$. This will be the manifestation of the so-called "technical hypothesis" of earlier papers (for example, [17], [6], [15]) in the present context.

MaIn Theorem. Suppose that $\phi, G, y_{1}, \ldots, y_{l}$, and $K$ are as above. Suppose that for all $S$ sufficiently large, if $\max _{i=1, \ldots, l}\left|s_{i}\right| \leq S$, then

$$
\left|y_{1} s_{1}+\cdots+y_{l} s_{l}\right|>\exp (-S \log S) .
$$

Put

$$
\delta=\frac{l+d_{1}+2 d_{2}}{d}
$$

and assume that $\delta>2$ if $d_{2} \neq 0$ and Condition 1 below holds. If $l>\delta$, then

where $\llbracket x \rrbracket$ denotes the integer part of $x$.

$$
\text { trans } \operatorname{deg}_{Q} K \geq \llbracket \frac{l}{\delta} \rrbracket
$$

In Section 3 below we will show how inequalities of the type (1) imply that Condition 1 holds in many interesting applications, such as for values of the unusual exponential function of Weierstrass elliptic functions. Similar applications have already been studied by the present author in [15]. The main theorem above appears to be at the limit of the present method and Condition 1 below is indispensable for the application of modern algebraic independence criteria.

\section{The technical hypothesis}

We begin by prescribing an embedding of $G$ into multiprojective space. Let $G_{2} \stackrel{f}{\rightarrow} \mathbb{P}_{N}$ be the embedding of $G_{2}$ into projective $N$-space as described 
by J-P. Serre in [13]. Then $G$ has the natural embedding into multiprojective space

$$
G \rightarrow \mathbb{P}_{d_{0}} \times \mathbb{P}_{d_{1}} \times \mathbb{P}_{N}
$$

In the interest of minimizing our notation we take $\phi(z)$ to denote the original analytic homomorphism $\phi: \mathbb{C} \rightarrow G(\mathbb{C})$ composed with this embedding. Then $\phi(z)$ may be represented as

$$
\phi(z)=\left(1, \alpha_{d_{0}} z: 1, e^{\beta_{1} z}, \ldots, e^{\beta_{d_{1}} z}: h_{0}(z), \ldots, h_{N}(z)\right),
$$

where $\alpha_{d_{0}}, \beta_{1}, \ldots, \beta_{d_{1}}$ are complex numbers and

$$
h_{0}(z), \ldots, h_{N}(z)
$$

are entire functions of order of growth at most two. Of course if $d_{0}=0$ then the coordinates corresponding to $\mathbb{P}_{d_{0}}$ are missing in the description of $\phi(z)$ above. Similarly if $d_{1}=0$ or $d_{2}=0$ the corresponding coordinates are deleted.

Let $\theta_{1}, \ldots, \theta_{r}$ be a set of generators for $K$ over $\mathbb{Q}$, with $\theta_{1}, \ldots, \theta_{t}$, $t \leq r$, a transcendence basis for $K$ over $\mathbb{Q}$ and suppose that $\mathscr{J}$ is the ideal of all polynomials in $\mathbb{C}\left[x_{1}, \ldots, x_{r}\right]$ vanishing at $\boldsymbol{\theta}=\left(\theta_{1}, \ldots, \theta_{r}\right)$. So the dimension of $\mathcal{J}$ is $t$. Furthermore, as each $\phi\left(y_{i}\right) \in G(K) \subseteq \mathbb{P}_{d_{0}} \times \mathbb{P}_{d_{i}} \times \mathbb{P}_{N}(K)$ we have multiprojective coordinates for $\phi\left(y_{i}\right)$ given by

$$
P_{i}=\left(a_{0, i}(\boldsymbol{\theta}), a_{d_{0}, i}(\boldsymbol{\theta}): m_{0, i}(\boldsymbol{\theta}), \ldots, m_{d_{1}, i}(\boldsymbol{\theta}): g_{0, i}(\boldsymbol{\theta}), \ldots, g_{N, i}(\boldsymbol{\theta})\right),
$$

where $a_{0, i}, \ldots, g_{N, i}$ are polynomials in $\mathbb{Z}\left[X_{1}, \ldots, X_{r}\right]$ of size at most $c_{1}$. (The lower case constants $c_{1}, c_{2}, \ldots$ which appear throughout this paper depend at most on $G, f, \phi, y_{1}, \ldots, y_{l}$, and our choice of generators $\left.\theta_{1}, \ldots, \theta_{r}.\right)$

Proposition 1. There exists a Zariski open set $\Omega \subseteq \mathbb{C}^{t}$ such that for all

$$
\tilde{\boldsymbol{\theta}}=\left(\tilde{\theta}_{1}, \ldots, \tilde{\theta}_{r}\right) \in \mathbb{C}^{r} \cap Z(\mathscr{J}),
$$

with $\left(\tilde{\theta}_{1}, \ldots, \tilde{\theta}_{t}\right) \subseteq \Omega$, all of the following hold.

(a) There exists a commutative algebraic group,

$$
\widetilde{G}=\mathbb{G}_{a}^{d_{0}} \times \mathbb{G}_{m}^{d_{1}} \times \widetilde{G}_{2},
$$

with $\tilde{G}_{2}$ of dimension $d_{2}$ which is defined over

$$
\widetilde{K}=\mathbb{Q}\left(\tilde{\theta}_{1}, \ldots, \tilde{\theta}_{r}\right) .
$$

(b) For each $i, 1 \leq i \leq l$, let $\widetilde{P}_{i}$ denote the point obtained from $P_{i}$ by replacing $\boldsymbol{\theta}$ by $\tilde{\theta}$ in each coordinate of $P_{i}$ when it is represented as in (3). 
Let $\tilde{\Gamma}=\widetilde{P}_{1} \mathbb{Z}+\cdots+\widetilde{P}_{l} \mathbb{Z}$ where $\widetilde{P}$ is deleted from the list of generators $\tilde{\Gamma}$ if $\widetilde{P}_{i} \notin \mathbb{P}_{d_{0}} \times \mathbb{P}_{d_{1}} \times \mathbb{P}_{N}$. Then $\tilde{\Gamma}$ is a finitely generated subgroup of $\tilde{G}(\widetilde{K})$.

(c) Let $\mathscr{P}$ denote the set of points among $P_{1}, \ldots, P_{l}$ for which $\widetilde{P}_{i} \in \mathbb{P}_{d_{0}} \times$ $\mathbb{P}_{d_{1}} \times \mathbb{P}_{N}$. Then the mapping $\sum_{P \in \mathscr{P}} m_{i} P_{i} \mapsto \sum_{P \in \mathscr{P}} m_{i} \widetilde{P}_{i}$ is a homomorphism from $\Gamma$ to $\tilde{\Gamma}$.

Proof. Let $R$ denote the ring $\mathbb{Z}\left[\theta_{1}, \ldots, \theta_{r}\right]$. By Proposition 5 of [7] there exists $\alpha \in R$ such that for any homomorphism $\psi: R \rightarrow \mathbb{C}$ with $\psi(\alpha) \neq 0$, there exists an algebraic group $G_{\psi}$, of dimension $d_{2}$, which is defined over the field of fractions of $\mathbb{Z}\left[\psi\left(\theta_{1}\right), \ldots, \psi\left(\theta_{r}\right)\right]$ (which we denote by $K_{\psi}$ ). Hence we only need to verify that we can find such a homomorphism taking $\theta_{i}$ to $\tilde{\theta}_{i}$ for $1 \leq i \leq r$.

Recall that we have assumed that $\theta_{1}, \ldots, \theta_{t}$ is a transcendence basis for $K$ over $\mathbb{Q}$. Hence there exist non-zero polynomials

$$
R_{j}=u_{0, j}+\cdots+u_{d_{j}, j} X_{j}^{d_{j}} \in \mathbb{Z}\left[\theta_{1}, \ldots, \theta_{t}\right]\left[X_{j}\right], \quad t+1 \leq j \leq r,
$$

such that $R_{j}\left(\theta_{j}\right)=0$. Additionally since $\alpha$ mentioned above lies in $\mathbb{Z}\left[\theta_{1}, \ldots, \theta_{r}\right]$ there exists a non-zero polynomial $q=v_{0}+\cdots+v_{d_{0}} X^{d_{0}}$ with $v_{i} \in \mathbb{Z}\left[\theta_{1}, \ldots, \theta_{t}\right]$ such that $q(\alpha)=0$. For $\tilde{\boldsymbol{\theta}}=\left(\tilde{\theta}_{1}, \ldots, \tilde{\theta}_{t}\right)$, with no $\tilde{\theta}_{i}=0$, at which none of $u_{0, j}, u_{d_{j}, j}(t+1 \leq j \leq r)$ nor $v_{0}, v_{d_{0}}$ vanishes, the mapping $\psi: R \rightarrow \mathbb{C}$ defined by $\psi\left(\theta_{i}\right)=\tilde{\theta}_{i}, 1 \leq i \leq r$, is a homomorphism with $\psi(\alpha) \neq 0$. This establishes part (a) of the proposition where the Zariski open set is the complement of $v_{0} v_{d_{0}} \prod_{j=t+1}^{r} u_{0, j} u_{d_{j, j}, \prod_{i=1}^{t}} x_{i}=0$.

Parts (b) and (c) then follow directly from part (2) of [7, Proposition 5].

The set $\Omega$ depends effectively on $G, \boldsymbol{\theta}$, and $f$. Thus, as $\left(\theta_{1}, \ldots, \theta_{t}\right) \in$ $\Omega$, there exists $r_{0}>0$ (effectively computable in terms of $G, \theta$, and $f$ ) such that $\left\{\mathbf{z}: \mathbf{z} \in \mathbb{C}^{t},\left|\mathbf{z}-\left(\theta_{1}, \ldots, \theta_{t}\right)\right|<r_{0}\right\} \subseteq \Omega$. We will also use the notation

$$
B(\theta, R)=\left\{\mathbf{z}: \mathbf{z} \in \mathbb{C}^{r},|\mathbf{z}-\theta|<R\right\} .
$$

To state our hypothesis we need an additional bit of notation. When $V_{1}, \ldots, V_{p}$ are subvarieties of $\mathbb{P}_{N_{1}}, \ldots, \mathbb{P}_{N_{p}}$ respectively, let

$$
H\left(V_{1} \times \cdots \times V_{p} ; D_{1}, \ldots, D_{p}\right)=\frac{\left(\sum_{i=1}^{p} \operatorname{dim} V_{i}\right) !}{\prod_{i=1}^{p}\left(\operatorname{dim} V_{i}\right) !} \prod_{i=1}^{p}\left(D_{i}^{\operatorname{dim} V_{i}} \operatorname{deg} V_{i}\right),
$$

where $\operatorname{deg} V_{i}$ denotes the degree of $V_{i}$, that is, the number of points of intersection of $V_{i}$ with $\operatorname{dim} V_{i}$ generic hyperplanes. $H\left(V_{1} \times \cdots \times V_{p} ; D_{1}, \ldots, D_{p}\right)$ 
is the homogeneous polynomial equal to $\operatorname{dim}\left(V_{1} \times \cdots \times V_{p}\right)$ ! times the homogeneous part of degree $\operatorname{dim}\left(V_{1} \times \cdots \times V_{p}\right)$ of the Hilbert-Samuel polynomial of $V \times \cdots \times V_{p}$.

Let $\rho=d_{0} / d$. Then for any real number $S>1$ define real numbers $L_{0}, L_{1}, D, \Sigma, \Delta$, and $\Delta_{1}$ by

(4) $c(G)^{1 / d} S^{\delta}(\log S)^{\rho}=\Sigma=L_{0}(\log S)=L_{1} S=D S^{2}, S^{l} \log S=\Delta_{1}=\Delta^{\delta / 2}$,

where

$$
c^{-1}(G)=\frac{d ! 2^{d_{2}}}{d_{0} ! d_{1} ! d_{2} !} \operatorname{deg} G_{2} .
$$

We also let $\mathbb{Z}(S)=\{n: 0 \leq n<S\}$ and put $\widetilde{\Gamma}(S)=\widetilde{P}_{1} \mathbb{Z}(S)+\cdots+\widetilde{P}_{l} \mathbb{Z}(S)$. Recall that a subgroup $H$ of an algebraic subgroup $G$ is incompletely defined in $G$ by equations of multidegree $\left(D_{0}, D_{1}, D_{2}\right)$ if the ideal of $H$ is generated modulo the ideal of $G$ by polynomials of multidegree at most $\left(D_{0}, D_{1}, D_{2}\right)$.

We can now state our "technical hypothesis."

Condition 1. There exists $S_{0}>0$ such that for all $S>S_{0}$ the following holds. For all but finitely many $\tilde{\boldsymbol{\theta}} \in \boldsymbol{B}(\boldsymbol{\theta}, \exp (-\Delta)) \cap \mathbb{Z}(\mathscr{J})$, as well as for $\tilde{\boldsymbol{\theta}}=\boldsymbol{\theta}$ and for all connected algebraic subgroups $\widetilde{G}^{\prime}$ of $\widetilde{G}$ with $\operatorname{dim} \widetilde{G}^{\prime}<$ $\operatorname{dim} \widetilde{G}$, which are incompletely defined in $\widetilde{G}$ by equations of multidegree at most $\left(L_{0}, L_{1}, 2 D\right)$, we have

$$
\operatorname{card}\left(\frac{\widetilde{\Gamma}(S)+\widetilde{G}^{\prime}}{\widetilde{G}^{\prime}}\right) \geq \frac{H\left(\widetilde{G} ; L_{0}, L_{1}, 2 D\right)}{H\left(\widetilde{G}^{\prime} ; L_{0}, L_{1}, D\right)} .
$$

As indicated above, we verify in Section 3 below that Condition 1 holds under rather mild assumptions in several classical cases.

\section{Proof of the main theorem}

The outline of our proof is straightforward. Suppose that $G$ is defined over $\mathbb{Q}\left(\theta_{1}, \ldots, \theta_{r}\right)$ and let $\theta$ be the $k=r+\left(d_{0}+d_{1}+N\right) l$-tuple.

$$
\begin{gathered}
\boldsymbol{\theta}=\left(\theta_{1}, \ldots, \theta_{r}, \alpha_{d_{0}} y_{1}, e^{\beta_{1} y_{1}}, \ldots, e^{\beta_{d_{1}} y_{1}}, h_{0}\left(y_{1}\right), \ldots, h_{N}\left(y_{1}\right), \ldots,\right. \\
\left.\alpha_{d_{0}} y_{l}, e^{\beta_{1} y_{l}}, \ldots, e^{\beta_{d_{1}} y_{l}}, h_{0}\left(y_{l}\right), \ldots, h_{N}\left(y_{l}\right)\right) .
\end{gathered}
$$

Under the assumption that our theorem is false we construct an ideal $\mathbf{I}(S)$ in $\mathbb{Z}\left[x_{1}, \ldots, x_{k}\right]$ of dimension $t$, which vanishes at $\boldsymbol{\theta} \in \mathbb{C}^{k}$. We then augment $I(S)$ so that it no longer vanishes at $\theta$ or at any points in a small neighborhood of $\theta$, but such that the new ideal is small at $\theta$ (that is, its 
generators have a small modulus when evaluated at $\theta$ ). To guarantee the non-vanishing of $I(S)$ near (and at) $\theta$ we invoke the technical condition (Condition 1) and a multiplicity estimate for algebraic groups. We repeat this process and construct a sequence of such ideals $\mathbf{I}(S)\}_{S \geq S_{0}}$, which by a criterion for algebraic independence due to P. Philippon [10] implies the algebraic independence result we desire.

To begin, we need explicit multiprojective coordinates for the points

$$
P^{\mathbf{s}}=\phi\left(s_{1} y_{1}+\cdots+s_{l} y_{l}\right),
$$

for $\mathbf{s}=\left(s_{1}, \ldots, s_{l}\right) \in \mathbb{Z}^{l}$, in terms of the multiprojective coordinates for $P_{i}=\phi\left(y_{i}\right), 1 \leq i \leq l$. This was done in [15] but we include these details here for clarity. The group laws on $\mathbb{G}_{a}$ and $\mathbb{G}_{m}$ are explicit and on $G_{2}$ the group law can at least be described. Recalling the representation of $P_{i}$ given by (3), we note that $\left(a_{0, i}(\boldsymbol{\theta}), s_{i} a_{d_{0}, i}(\boldsymbol{\theta})\right)$ are $\mathbb{G}_{a}^{d_{0}}$-coordinates for $\phi\left(s_{i} y_{i}\right)$. Since the group law on $\mathbb{G}_{a}$ is given by $\left(x_{1}, y_{1}\right)+\left(x_{2}, y_{2}\right)=\left(x_{1} x_{2}, x_{1} y_{2}+x_{2} y_{1}\right)$ one establishes by induction that $\mathbb{G}_{a}^{d_{0}}$-coordinates of $P^{\mathbf{s}}$ may be given as

$$
\left(U_{0, \mathbf{s}}(\boldsymbol{\theta}), U_{d_{0}, \mathbf{s}}(\boldsymbol{\theta})\right) \text {, }
$$

where

$$
U_{0, \mathbf{s}}(\mathbf{x})=\prod_{i=1}^{l} a_{0, i}(\mathbf{x})
$$

and

$$
U_{d_{0}, \mathbf{s}}(\mathbf{x})=\sum_{i=1}^{l}\left(s_{i} a_{d_{0}, i}(\mathbf{x}) \prod_{j \neq i} a_{0, i}(\mathbf{x})\right) .
$$

The group law on $\mathbb{G}_{m}^{d_{1}}$ is even easier to describe. Again recalling the representation (3) for the points $P_{i}, 1 \leq i \leq l$, one sees that

$$
\left(M_{0, \mathbf{s}}(\boldsymbol{\theta}), \ldots, M_{d_{1}, \mathbf{s}}(\boldsymbol{\theta})\right) \text {, }
$$

with

$$
M_{j, \mathbf{s}}(\mathbf{x})=\prod_{i=1}^{l} m_{j, i}^{s_{i}}(\mathbf{x}), \quad 0 \leq j \leq d_{1},
$$

are $\mathbb{G}_{m}^{d_{1}}$ coordinates for $P^{\mathbf{s}}$.

Finally we must exhibit $G_{2}$-coordinates for $P^{s}$. Let $\mathbf{X}_{j}$ denote the set of homogeneous variables $X_{j_{0}}, \ldots, X_{j_{N}}$ for $0 \leq j \leq l$. In [14, Proposition 2.2], we established that for $s \in \mathbb{Z}^{l}(S)$ there exist multihomogeneous polynomials $F_{k, \mathbf{s}}\left(\mathbf{X}_{1}, \ldots, \mathbf{X}_{l}\right) \quad 0 \leq k \leq N$, of degree at most $c_{2} S^{2}$, with coefficients in $\mathbb{Z}[\boldsymbol{\theta}]$ having size at most $c_{3} S^{2}$, such that

$$
\left(F_{0, \mathbf{s}}\left(\mathbf{h}\left(y_{1}\right), \ldots, \mathbf{h}\left(y_{l}\right)\right), \ldots, F_{N, \mathbf{s}}\left(\mathbf{h}\left(y_{1}\right), \ldots, \mathbf{h}\left(y_{l}\right)\right)\right)
$$


are projective coordinates for the projection of $P^{\mathbf{s}}$ onto $G_{2}$. Rewriting each $h_{j}\left(y_{i}\right)$ as an element of $\mathbb{Z}[\theta]$ we see that for each $k, 0 \leq k \leq n$, and for each $s \in \mathbb{Z}^{l}(S)$ there exist polynomials $A_{k, \mathbf{s}} \in \mathbb{Z}[\mathbf{X}]$ of size at most $c_{4} S^{2}$, such that the projective coordinates for the projection of $P^{s}$ onto $G_{2}$ are given by

$$
\left(A_{0, \mathbf{s}}(\boldsymbol{\theta}), \ldots, A_{N, \mathbf{s}}(\boldsymbol{\theta})\right)
$$

Thus

$$
\left(U_{0, \mathbf{s}}(\boldsymbol{\theta}), U_{d_{0}, \mathbf{s}}(\boldsymbol{\theta}): M_{0, \mathbf{s}}(\boldsymbol{\theta}), \ldots, M_{d_{1}, \mathbf{s}}(\boldsymbol{\theta}): A_{0, \mathbf{s}}(\boldsymbol{\theta}), \ldots, A_{N, \mathbf{s}}(\boldsymbol{\theta})\right)
$$

are multiprojective coordinates for $P^{\mathbf{s}}$.

Failing to have lower bounds for the coordinates $\left|A_{k, \mathrm{~s}}(\theta)\right|$, which we need at a later stage, we now employ an idea from [16]. If for some $s \in \mathbb{Z}^{l}(S)$ we have

$$
\max _{1 \leq k \leq N}\left|A_{k, \mathbf{s}}(\boldsymbol{\theta})\right|<e^{-\Delta / 2}
$$

put

$$
I(S)=\left(A_{0, \mathbf{s}}(\mathbf{x}), \ldots, A_{N, \mathbf{s}}(\mathbf{x})\right) .
$$

Then $\theta$ is not a zero of $I(S)$ since $\left(A_{0, \mathrm{~s}}(\theta), \ldots, A_{N, \mathbf{s}}(\theta)\right)$ is a point in projective space. Similarly, if $\tilde{\theta} \in B(\theta, \exp (-\Delta))$ is a zero of $I(S)$ then the projection of the point

$$
\widetilde{P}^{\mathbf{s}}=s_{1} \widetilde{P}_{1}+\cdots+s_{l} \widetilde{P}_{l}
$$

onto $G_{2}$ does not lie in projective space. However, Condition 1 insures that for all but finitely many such $\tilde{\boldsymbol{\theta}}$ 's,

$$
\operatorname{card}(\tilde{\Gamma}(S)) \geq c^{-1}(G) L_{0}^{d_{0}} L_{1}^{d_{1}} D^{d_{2}} \geq S^{l} .
$$

Thus $\widetilde{P}_{i}$ lies in $\widetilde{G}_{2}(\widetilde{K})$ for $1 \leq i \leq l$ and $\widetilde{P}^{\mathbf{s}}$ is therefore an element of projective space. As before we deduce that $I(S)$ has only finitely many zeros in $B(\theta, \exp (-\Delta))$.

Henceforth we assume that (6) does not hold. Let $Z, Y$ and $X$ denote the families of homogeneous variables $\left\{Z_{0}, Z_{d_{0}}\right\},\left\{Y_{0}, \ldots, Y_{d_{1}}\right\}$ and $\left\{X_{0}, \ldots, X_{N}\right\}$. Let $\left\{\mathscr{M}_{i}: i \in I\right\}$ be a set of multihomogeneous monomials, which are homogeneous of degree $L_{0}$ in $\mathrm{Z}$, homogeneous of degree $L_{1}$ in $\mathbf{Y}$, homogeneous of degree $D$ in $\mathbf{X}$ and such that this set is a maximal with respect to the property that its elements are linearly independent modulo the ideal defining $G$ in multiprojective space. Put

$$
c^{*}=\frac{\operatorname{card}(I)}{L_{0}^{d_{0}} L_{1}^{d_{1}} D^{d_{2}}} .
$$


Lemma 1. Let $S_{0}=\left(c^{*} / 2^{k-1}\right)^{1 / l} S$. There exist polynomials $q_{\mathbf{i}} \in \mathbb{Z}[\mathbf{x}]$ for $\mathbf{i}=(\mathbf{e}, \mathbf{f}, \mathbf{g}) \in I$, not all zero, of degree at most $\Sigma$ and of logarithmic height at most $c_{5} \Sigma$ such that for $\mathbf{s} \in \mathbb{Z}^{l}\left(S_{0}\right)$

$$
Q_{\mathbf{s}}(\mathbf{x})=\sum_{\mathbf{i} \in I} q_{\mathbf{i}}(\mathbf{x})\left(\prod_{t=0}^{d_{0}} U_{t, \mathbf{s}}^{e_{t}}(\mathbf{x}) \prod_{r=0}^{d_{1}} M_{r, \mathbf{s}}^{f_{r}}(\mathbf{x}) \prod_{k=0}^{N} A_{k, \mathbf{s}}^{g_{k}}(\mathbf{x})\right)
$$

is identically zero.

Proof. This is a direct application of Siegel's lemma (the box principle). View the coefficients of the polynomials $q_{i}(\mathbf{x})$ as unknowns, there are $\operatorname{card}(I)(1+\Sigma)^{k}$ of these. For each $\mathbf{s} \in \mathbb{Z}^{l}\left(S_{0}\right)$ rewrite the equation $Q_{\mathbf{s}}(\mathbf{x})=0$ as a system of at most $(1+2 \Sigma)^{k}$ equations by setting the coefficient of each monomial in $x_{1}, \ldots, x_{k}$ equal to zero. This yields a system of $S_{0}^{l}(1+2 \Sigma)^{k}$ equations. By our choice of parameters $L_{0}, L_{1}, D$ this system has a nontrivial solution with coefficients lying in $\mathbb{Z}$.

We now use an idea attributed to $G$. Chudnovsky to alter the coefficient polynomials $q_{\mathbf{i}}, \mathbf{i} \in I$, so that they have no common zero in $B\left(\theta, \exp \left(-\Delta_{1}\right)\right)$. For $\mathbf{j}=\left(j_{1}, \ldots, j_{k}\right)$. denote the differential operator $\partial^{j_{1}+\cdots+j_{k}} / \partial x_{1}^{j_{1}} \cdots \partial x_{k}^{j_{k}}$ by $\partial^{j}$. For a point $\tilde{\boldsymbol{\theta}} \in \mathbb{C}^{k}$ there exists $\mathbf{j}$ with $\max _{1 \leq i \leq k} j_{i} \leq c_{6} \Sigma$ such that not all of $q_{\mathbf{i}, \mathbf{j}}=\left(1 / j_{1} ! \cdots j_{k} !\right) \partial^{\mathbf{j}} q_{\mathbf{i}}, \mathbf{i} \in I$, vanish at $\tilde{\boldsymbol{\theta}}$.

Choose $\mathbf{j}(\tilde{\boldsymbol{\theta}}) \in \mathbb{N}^{k}$ with $\|\mathbf{j}(\tilde{\boldsymbol{\theta}})\|=j_{1}+\cdots+j_{k}$ minimal such there exists $\mathbf{i} \in I$ with $q_{\mathbf{i}} \neq 0$ and $\partial^{\mathbf{i}(\theta)} q_{\mathbf{i}}(\tilde{\boldsymbol{\theta}}) \neq 0$. (Hence for $\mathbf{j} \in \mathbb{N}^{k}$ with $\|\mathbf{j}\|<$ $\|\mathbf{j}(\tilde{\boldsymbol{\theta}})\|, \partial_{q_{\mathbf{i}}}^{\mathbf{j}}(\tilde{\boldsymbol{\theta}})=0$ for all $\mathbf{i} \in I$.) Put $J_{\Delta}=\left\{\mathbf{j}(\tilde{\boldsymbol{\theta}}): \tilde{\boldsymbol{\theta}} \in B\left(\boldsymbol{\theta}, \exp \left(-\Delta_{1}\right)\right)\right\}$.

For $\mathbf{s} \in \mathbb{Z}^{l}\left(S_{1}\right)$ with $S_{1}=c_{7} S, c_{7}>1$ and $\mathbf{j} \in J_{\Delta}$ put

$$
Q_{\mathbf{s}}(\mathbf{x})=\sum_{\mathbf{i} \in I}(\mathbf{x})\left(\prod_{t=0}^{d_{0}} U_{t, \mathbf{s}}^{e_{t}}(\mathbf{x}) \prod_{r=0}^{d_{1}} M_{r, \mathbf{s}}^{f_{r}}(\mathbf{x}) \prod_{k=0}^{N} A_{k, \mathbf{s}}^{g_{k}}(\mathbf{x})\right) .
$$

Put $\mathscr{J}^{\prime}\left(S_{1}\right)=\left(Q_{\mathbf{s j}}(\mathbf{x}), \mathscr{J}\right)_{\mathbf{s} \in \mathbf{Z}^{\prime}\left(S_{1}\right)}$.

Lemma 2. The ideal $\mathcal{J}^{\prime}\left(S_{1}\right)$ has only finitely many zeros in the ball $B\left(\boldsymbol{\theta}, \exp \left(-\Delta_{1}\right)\right) \subseteq \mathbb{C}^{k}$ and does not vanish at $\boldsymbol{\theta}$.

Proof. Suppose $\tilde{\theta} \in B\left(\theta, \exp \left(-\Delta_{1}\right)\right)$ is a zero of the ideal $\mathscr{J}^{\prime}\left(S_{1}\right)$. Consider the multihomogeneous polynomial

$$
P_{j(\tilde{\theta})}(\mathbf{Z}, \mathbf{Y}, \mathbf{X})=\sum_{\mathbf{i} \in I} q_{\mathbf{i j}(\tilde{\theta})}(\tilde{\boldsymbol{\theta}}) \mathscr{M}_{\mathrm{i}},
$$


where we recall the monomials $\mathscr{M}_{\mathbf{i}}, \mathbf{i} \in I$, from above. By our choice of $\mathbf{j}(\tilde{\boldsymbol{\theta}})$ this is not the zero polynomial. Since $\tilde{\boldsymbol{\theta}}$ is a zero of $\mathscr{J}^{\prime}\left(S_{1}\right), Q_{\mathrm{sj}(\tilde{\theta})}(\tilde{\theta})=0$ for $\mathbf{s} \in \mathbb{Z}^{l}\left(S_{1}\right)$. Thus the polynomial $P_{\mathbf{j}(\tilde{\theta})}(\mathbf{Z}, \mathbf{Y}, \mathbf{X})$ vanishes along $\phi(z)$ on $\widetilde{\Gamma}\left(S_{1}\right)=\left\{s_{1} \widetilde{P}_{1}+\cdots+s_{l} \widetilde{P}_{l}: 0 \leq s_{i} \leq S_{1}\right\}$.

Let $\widetilde{G}$ denote the algebraic group which is associated with $\tilde{\theta}$ by Proposition 1. We now apply the zeros estimate of $P$. Philippon, [11, Théorème 2.1], to conclude that there exists a connected algebraic subgroup $\widetilde{G}^{\prime}$ of $\widetilde{G}$, which is incompletely defined by multihomogeneous equations of multidegree at most $\left(L_{0}, L_{1}, 2 D\right)$, such that

$$
\operatorname{card}\left(\frac{\widetilde{\Gamma}\left(S_{1}\right)+\widetilde{G}^{\prime}}{\widetilde{G}}\right) \leq \frac{H\left(\widetilde{G} ; L_{0}, L_{1}, 2 D\right)}{H\left(\widetilde{G}^{\prime} ; L_{0}, L_{1}, D\right)} .
$$

If we let $L_{0}^{\prime}, L_{1}^{\prime}$ and $D^{\prime}$ be the real numbers associated with $S_{1}$ by the relation (4) then

$$
\frac{H\left(\widetilde{G} ; L_{0}, L_{1}, 2 D\right)}{H\left(\widetilde{G}^{\prime} ; L_{0}, L_{1}, D\right)}<\frac{H\left(\widetilde{G} ; L_{0}^{\prime}, L_{1}^{\prime}, 2 D^{\prime}\right)}{H\left(\widetilde{G}^{\prime} ; L_{0}^{\prime}, L_{1}^{\prime}, D^{\prime}\right)},
$$

which together with (8) contradicts Condition 1 unless $\tilde{\theta}$ belongs to the finite set of zeros allowed by Condition 1. This establishes Lemma 2.

We next estimate $\left|Q_{\mathrm{s}}(\boldsymbol{\theta})\right|$ for $\mathbf{s} \in \mathbb{Z}^{l}\left(S_{1}\right)$ and $\mathbf{j} \in J_{\Delta}$. Here we follow the outline of a similar estimate given by G. Diaz [6] in the case of the ordinary exponential function. For each $\mathbf{i} \in I$ put

$$
M_{\mathbf{i}}(\mathbf{x})=\prod_{t=0}^{d_{0}} U_{t, \mathbf{s}}^{e_{t}}(\mathbf{x}) \prod_{r=0}^{d_{1}} M_{r, \mathbf{s}}^{f_{r}}(\mathbf{x}) \prod_{k=0}^{N} A_{k, \mathbf{s}}^{g_{k}}(\mathbf{x}) .
$$

Then for $\mathbf{s} \in \mathbb{Z}^{l}\left(S_{1}\right)$ and $\mathbf{j} \in J_{\Delta}$,

$$
Q_{\mathbf{s j}}(\theta)=\sum_{\mathbf{i} \in I} q_{\mathbf{i j}}(\theta) M_{\mathbf{i}}(\theta) .
$$

Therefore, if we put

$$
A_{\mathbf{s j}}(\boldsymbol{\theta})=\sum_{\mathbf{i} \in I}\left(q_{\mathbf{i j}}(\boldsymbol{\theta})-q_{\mathbf{i j}}(\tilde{\theta})\right) M_{\mathbf{i}}(\boldsymbol{\theta})
$$

and

$$
B_{\mathrm{sj}}(\boldsymbol{\theta})=\sum_{\mathbf{i} \in I} q_{\mathrm{ij}}(\tilde{\boldsymbol{\theta}}) M_{\mathbf{i}}(\boldsymbol{\theta}),
$$

where $\tilde{\boldsymbol{\theta}}$ is such that $\mathbf{j}=\mathbf{j}(\tilde{\boldsymbol{\theta}})$, then

$$
\left|Q_{\mathrm{sj}}(\boldsymbol{\theta})\right| \leq\left|A_{\mathrm{sj}}(\boldsymbol{\theta})\right|+\left|B_{\mathrm{sj}}(\boldsymbol{\theta})\right| .
$$


The estimate of $\left|A_{\mathbf{s j}}(\theta)\right|$ is the more straightforward of the two. Using the estimate

$$
\left|q_{\mathrm{ij}}(\boldsymbol{\theta})-q_{\mathrm{ij}}(\tilde{\boldsymbol{\theta}})\right|<|\boldsymbol{\theta}-\tilde{\boldsymbol{\theta}}| e^{c_{8} \Sigma}<e^{-c_{9} \Delta_{1}},
$$

provided $S$ is sufficiently large, we obtain

$$
\left|A_{\mathrm{sj}}(\theta)\right|<\operatorname{card}(I) e^{-c_{q} \Delta_{1}} \max _{i \in I}\left|M_{i}(\theta)\right|<e^{-c_{10} \Delta_{1}},
$$

again provided $S$ is sufficiently large. We note that this estimate holds for $\mathbf{s} \in \mathbb{Z}^{l}\left(S_{1}\right)$ possibly with a different constant.

We estimate $\left|B_{\mathrm{sj}}(\theta)\right|$ by taking

$$
B_{\mathbf{s j}}(\boldsymbol{\theta})=C_{\mathbf{s j}}(\tilde{\boldsymbol{\theta}})+D_{\mathbf{s j}}(\boldsymbol{\theta}),
$$

where

$$
C_{\mathbf{s j}}(\tilde{\boldsymbol{\theta}})=\sum_{\mathbf{i} \in I} q_{\mathbf{i j}}(\tilde{\boldsymbol{\theta}}) M_{\mathbf{i}}(\tilde{\boldsymbol{\theta}})
$$

and

$$
D_{\mathbf{s} \mathbf{j}}(\boldsymbol{\theta})=\sum_{\mathbf{i} \in I} q_{\mathrm{ij}}(\tilde{\boldsymbol{\theta}})\left(\boldsymbol{M}_{\mathbf{i}}(\boldsymbol{\theta})-\boldsymbol{M}_{\mathbf{i}}(\tilde{\boldsymbol{\theta}})\right) .
$$

We recall that by Lemma 1 we have $Q_{\mathbf{s}}(\mathbf{x})=0$, hence for $\mathbf{j} \in \mathbb{N}^{k}$ also $\partial^{\mathrm{j}} Q_{\mathbf{s}}$ is identically zero. But

$$
\partial^{\mathbf{i}} Q_{\mathbf{s}}(\mathbf{x})=\sum_{\mathbf{i} \in I} \sum_{\substack{\mathbf{j}^{\prime} \in \mathbf{N}^{k} \\
\mathbf{j}^{\prime \prime} \in \mathbb{N}^{k} \\
\mathbf{j}^{\prime}+\mathbf{j}^{\prime \prime}=\mathbf{j}}}\left(\begin{array}{c}
j_{1} \\
j_{1}^{\prime}
\end{array}\right) \cdots\left(\begin{array}{c}
j_{k} \\
j_{k}^{\prime}
\end{array}\right) \partial^{\mathbf{i}^{\prime}} q_{\mathbf{i}}(\mathbf{x}) \partial^{\mathbf{j}^{\prime \prime}} M_{\mathbf{i}}(\mathbf{x}) .
$$

By our choice of $\mathbf{j}=\mathbf{j}(\tilde{\boldsymbol{\theta}})$ we have

$$
\left.\partial^{\mathbf{j}^{\prime}} q_{\mathbf{i}}(\mathbf{x})\right|_{\mathbf{x}=\tilde{\boldsymbol{\theta}}}=0 \text { if }\left\|\mathbf{j}^{\prime}\right\|<\|\mathbf{j}\|
$$

and therefore

$$
\partial^{\mathrm{i}} Q_{\mathbf{s}}(\tilde{\boldsymbol{\theta}})=\sum_{\mathbf{i} \in I} q_{\mathrm{ij}}(\tilde{\boldsymbol{\theta}}) \boldsymbol{M}_{\mathbf{i}}(\tilde{\boldsymbol{\theta}})
$$

Thus $C_{\mathbf{s j}}(\tilde{\boldsymbol{\theta}})=0$.

To estimate $\left|D_{\mathrm{sj}}(\theta)\right|$ we begin with

$$
\left|M_{\mathbf{i}}(\boldsymbol{\theta})-M_{\mathbf{i}}(\tilde{\boldsymbol{\theta}})\right|<\exp \left(-\Delta_{1}+c_{11}\left(L_{0} \log S+L_{1} S+D S^{2}\right)\right)
$$

and from $\left|q_{\mathrm{ij}}(\tilde{\theta})\right|<\exp \left(c_{12}\left(L_{0} \log S+L_{1} S+D S^{2}\right)\right)$ we deduce that

$$
\left|D_{\mathbf{s j}}(\tilde{\theta})\right|<\exp \left(-c_{13} \Delta_{1}\right),
$$

provided $S$ is sufficiently large, for all $\mathbf{s} \in \mathbb{Z}^{l}(S)$. Putting together the above results establishes our estimate for $\left|Q_{\mathrm{sj}}(\theta)\right|$.

We must provide such an estimate when $\mathbf{s}$ lies in the larger set $\mathbb{Z}^{l}\left(S_{1}\right)$. For this we need the following interpolation lemma. 
LemMA 3. Let $R$ and $R_{1}$ be two real numbers with $2<R_{1}<R / 4$. Suppose that $f$ is a function which is analytic in the disc $|z| \leq R$. Suppose that $x_{1}, \ldots, x_{l}$ are complex numbers and suppose that the numbers

$$
n_{1} x_{1}+\cdots+n_{l} x_{l}, \quad n_{i} \in \mathbb{Z}\left(N_{i}\right),
$$

are pairwise distinct. Call the set of all those values $X$ and put

$$
\mu=\min \left(1,\left|n_{1} x_{1}+\cdots+n_{l} x_{l}\right|, n_{i} \in \mathbb{Z}\left(N_{i}-1\right)\right) .
$$

Then for all pairs of nonnegative integers $(r, t)$,

$$
\begin{array}{r}
\left|f^{(r)}\right|_{R_{1}} \leq r !\left\{2|f|_{R}\left(\frac{4 R_{1}}{R}\right)^{t N_{1} \cdots N_{l}}+\frac{2 N_{1} \cdots N_{l}}{R_{1}}\left(\frac{33 R_{1}}{\mu\left(N_{1} \cdots N_{l}\right)^{1 / i}}\right)^{t N_{1} \cdots N_{l-1}}\right. \\
\left.\times \max _{\substack{x \in X \\
0 \leq \tau \leq t-1}}\left|\frac{f^{(\tau)}(x)}{\tau !}\right|\right\},
\end{array}
$$

where $i=1$ if the points of $X$ are colinear and $i=2$ otherwise.

Proof. Apply the estimate of [5, Lemma 6] in the proof of [12, Lemma $4.5]$.

We now apply this result to the function

$$
\tilde{F}(z)=\sum_{\mathbf{i} \in I} q_{\mathbf{i j}}(\tilde{\boldsymbol{\theta}}) \phi_{\mathbf{i}}(z),
$$

where $\phi_{\mathrm{i}}(z)$ denotes the monomial $\mathscr{M}_{\mathrm{i}}$ composed with $\phi(z)$ when represented as in (2). $\widetilde{F}(z)$ is an entire function, which is not identically zero. We also let

$$
F(z)=\sum_{\mathbf{i} \in I} q_{\mathrm{ij}}(\boldsymbol{\theta}) \phi_{\mathrm{i}}(z) .
$$

We first show that for $\mathbf{s} \in \mathbb{Z}^{l}\left(S_{0}\right)$,

$$
\left|F\left(s_{\mathrm{i}} y_{1}+\cdots+s_{l} y_{l}\right)\right|<e^{-c_{14} \Delta_{1}} .
$$

This comes from re-examining the polynomials $Q_{\mathrm{sj}}(\theta)$. Recalling the representation of $\phi(z)$ given in (2) we know that $\phi\left(s_{1} y_{1}+\cdots+s_{l} y_{l}\right)$ has multiprojective coordinates as represented in (5). We focus on each of the $\mathbb{G}_{a}, \mathbb{G}_{m}$ and $G_{2}$ coordinates for this point.

For $G_{2}$ we have

$$
\lambda_{2}\left(h_{0}(\mathbf{s} \cdot \mathbf{y}), \ldots, h_{N}(\mathbf{s} \cdot \mathbf{y})\right)=\left(A_{0, \mathbf{s}}(\boldsymbol{\theta}), \ldots, A_{N, \mathbf{s}}(\boldsymbol{\theta})\right)
$$


for $\lambda_{2} \in \mathbb{C} \backslash\{0\}$. By the theory of the theta functions we have $e^{-c_{15} S^{2}}<$ $\max _{1 \leq k \leq N}\left|h_{k}(\mathbf{x} \cdot \mathbf{y})\right| \leq e^{c_{16} S^{2}}$. Therefore, taking

$$
\lambda_{2}=A_{k, \mathbf{s}}(\boldsymbol{\theta}) / h_{k}(\mathbf{s} \cdot \mathbf{y})
$$

and recalling our assumption that (6) does not hold, we have

$$
e^{-c_{17} \max \left\{\Delta, S^{2}\right\}} \leq\left|\lambda_{2}\right| \leq c_{18} e^{c_{19} S^{2}},
$$

having employed the upper bound, $\left|A_{k, \mathrm{~s}}(\theta)\right| \leq c_{20} e^{c_{21} s^{2}}$.

On $\mathbb{G}_{a}$ and $\mathbb{G}_{m}$ the analogous estimates are more straightforward. For $\mathbb{G}_{a}$,

$$
\lambda_{a}\left(1, \alpha_{d_{0}}(\mathbf{s} \cdot \mathbf{y})\right)=\left(U_{0, \mathbf{s}}(\boldsymbol{\theta}), U_{d_{0}, \mathbf{s}}(\boldsymbol{\theta})\right)
$$

with $\lambda_{a}=U_{0, \mathbf{s}}(\boldsymbol{\theta})$ and therefore $\left|\lambda_{a}\right| \leq c_{22}$. For $\mathbb{G}_{m}$,

$$
\lambda_{m}\left(1, e^{\beta_{1}(\mathbf{s} \cdot \mathbf{y})}, \ldots, e^{\beta_{d_{1}}(\mathbf{s} \cdot \mathbf{y})}\right)=\left(M_{0, \mathbf{s}}(\boldsymbol{\theta}), \ldots, M_{d_{1}, \mathbf{s}}(\boldsymbol{\theta})\right),
$$

with $c_{23}^{\min s_{i}} \leq\left|\lambda_{m}\right| \leq c_{23}^{\max s_{i}}$.

However we then have

$$
\lambda_{a}^{L_{0}} \lambda_{m}^{L_{1}} \lambda_{2}^{D} F\left(s_{1} y_{1}+\cdots+s_{l} y_{l}\right)=Q_{\mathbf{s}, \mathrm{j}}(\boldsymbol{\theta})
$$

and therefore (11) holds.

But for all $z,|\widetilde{F}(z)| \leq|\widetilde{F}(z)-F(z)|+|F(z)|$ and therefore

$$
\left|\widetilde{F}\left(s_{1} y_{1}+\cdots+s_{l} y_{l}\right)\right| \leq\left|\sum_{\mathbf{i} \in I}\left(q_{\mathbf{i}}(\tilde{\theta})-q_{\mathbf{i}}(\boldsymbol{\theta})\right) \phi_{\mathbf{i}}\left(s_{1} y_{1}+\cdots+s_{l} y_{l}\right)\right| \leq c_{24} e^{-c_{25} \Delta_{1}} .
$$

We apply the above lemma to $\widetilde{F}(z)$ with $r=0, t=1, x_{i}=y_{i}, N_{i}=$ $S, R_{1}=S_{1}$ and $R=S_{1}^{1+\varepsilon}$, for a positive $\varepsilon<\frac{1}{2}(l-\delta)$ and

$$
\mu>\exp \left(-S_{1} \log S\right) \text {. }
$$

That this lower bound on $\mu$ holds follows from our assumption (1). Then we obtain

$$
|\widetilde{F}|_{S_{1}}<c_{26}|\widetilde{F}|_{R}\left(\frac{4}{S_{1}^{\varepsilon}}\right)^{S^{l}}+c_{27} \frac{S^{l}}{S_{1}}\left(\frac{c_{28} S_{1}}{\mu S^{l}}\right)^{S^{l-1}}\left(c_{24} e^{-c_{25} \Delta_{1}}\right) .
$$

But

$$
|\widetilde{F}|_{R}<\operatorname{card}(I) \max _{\mathrm{i} \in I}\left|q_{\mathrm{ij}}(\tilde{\boldsymbol{\theta}})\right| \max _{\mathrm{i} \in I}\left|\phi_{\mathrm{i}}\right|_{R}<\exp \left(c_{29} D R^{2}\right)
$$

and therefore by the estimate above for $\mu$,

$$
\log |\widetilde{F}|_{S_{1}}<-c_{30} S^{l} \log (S) \text {. }
$$


Hence for $s \in \mathbb{Z}^{l}\left(S_{1}\right)$,

$$
\left|B_{\mathrm{sj}}(\theta)\right|<\exp \left(-c_{31} S^{l} \log S\right) .
$$

Putting together (9) with the estimates (10) and (12) we have

$$
\left|Q_{\mathrm{sj}}(\theta)\right|<\exp \left(-c_{32} S^{l} \log S\right)
$$

for $\mathbf{s} \in \mathbb{Z}^{l}\left(S_{1}\right)$ and $\mathbf{j} \in J_{\Delta}$.

Thus if (6) holds for infinitely many choices of $S$ define a sequence of ideals $I(S)$ as in (7). Otherwise, for all $S$ sufficiently large let $I(S)=$ $\mathscr{J}^{\prime}\left(S_{1}\right)$.

In the former case we rename our parameters if necessary to obtain a sequence of ideals $I(N), N \geq N_{0}$, with generators of size at most $c_{4} N^{2}$ and of moduli at $\theta$ of at most $\exp \left(-N^{2 l / \delta}(\log N)^{1 / 2 \delta}\right)$, with no zeros within $\exp \left(-N^{2 l / \delta}(\log N)^{1 / 2 \delta}\right)$ of $\theta$. Then by [10, Theorème 2.11] there exists a constant $C$ (depending only on $G, y_{1}, \ldots, y_{l}, \phi$ ) such that

$$
N^{2 l / \delta}(\log N)^{1 / 2 \delta}<C N^{2(t+1)},
$$

from which it follows that $t \geq \llbracket l / \delta \rrbracket$.

In the latter case we obtain a sequence of ideals $I(N), N \geq N_{1}$, with generators of size at most $c_{33} \Sigma(N)$ and of moduli at most $\exp \left(-c_{32} N^{l}(\log N)\right)$ at $\boldsymbol{\theta}$. Moreover, $I(N)$ has at most finitely many zeros in $B\left(\theta, \exp \left(-N^{l}(\log N)\right)\right.$. Thus by [10, Théorème 2.11],

$$
c_{32} N^{l} \log N<C \Sigma^{l+1}=c_{34} N^{\delta(t+1)}(\log N)^{\rho(t+1)} .
$$

We first focus on the power of $\log N$ on both sides of this inequality. If $\rho(t+1) \geq 1$ then by our choice of $\rho$, we must have $d_{0}=1$ and $t \geq d \geq[l / \delta]$ which was to be shown. Otherwise, the main term exponents must satisfy the strict inequality $\delta(t+1)>l$, hence, $t \geq[l / \delta]$.

Thus we have established the main theorem.

\section{Applications}

In this section we explore several applications of our main theorem. Here we will study the algebraic independence of numbers associated with the usual exponential function, a Weierstrass elliptic function and points on a 1-dimensional analytic subgroup of an abelian variety.

Our first application is for the usual exponential function where we recover [6, Theorems 1 and 2] with marginally stronger hypotheses. 
THEOREM 1. Let $\left\{u_{1}, \ldots, u_{m}\right\}$ and $\left\{v_{1}, \ldots, v_{n}\right\}$ be two sets of complex numbers which are $\mathbb{Q}$-linearly independent.

(a) Let $\delta=(m+n) /(n+1)$. Suppose that there exists a real number $S_{0} \geq 0$ such that for all $s_{i} \in \mathbb{Z}(S), 1 \leq i \leq m$ and $t_{j} \in \mathbb{Z}(S), 1 \leq j \leq n$, with $S \geq S_{0}$ one has

$$
\left|\sum_{i=1}^{m} s_{i} u_{i}\right|>\exp \left(-\min \left(S \log S, S^{m /(\delta+1)}\right)\right)
$$

and

$$
\left|\sum_{j=1}^{n} t_{j} v_{j}\right|>\exp \left(-S^{m /(3 \delta-2)}\right) .
$$

If $m>\delta$ then trans $\operatorname{deg}_{\mathbb{Q}} \mathbb{Q}\left(u_{i}, e^{u_{i} v_{j}}: 1 \leq i \leq m, 1 \leq j \leq n\right) \geq[m / \delta]$.

(b) If the hypotheses of part (a) hold with $\delta=(m+n) / n$ then $m>\delta$ implies

$$
\text { trans } \operatorname{deg}_{\mathbb{Q}} \mathbb{Q}\left(e^{u_{i} v_{j}}: 1 \leq i \leq m, 1 \leq j \leq n\right) \geq\left[\frac{m}{\delta}\right] .
$$

We do not deduce this result from the main theorem but instead we refer the interested reader to [15] wherein a version of Theorem 1 demonstrating that the field under study has transcendence degree at least three (Corollary 1 ) is established. In fact Theorem 1 , as stated above, does not follow from our main theorem but from the main theorem with the ball $B(\theta, \exp (-\Delta))$ in Condition 1 replaced with the ball $B\left(\theta, \exp \left(-\Delta_{1}\right)\right)$. From the proof in Section 2 it follows that if our algebraic group is of the form $G=\mathbb{G}_{a}^{d_{0}} \times \mathbb{G}_{m}^{d_{1}}$ then the considerations we gave concerning the general group law on $G_{2}$ are not necessary and the main theorem holds with the (slightly) weakened Condition 1.

In the elliptic case our applications are new results. Let $E$ be an elliptic curve defined over $\overline{\mathbb{Q}}$ with associated Weierstrass function $\wp(z)$ and let $\sigma(z)$ denote its associated Weierstrass sigma function. Put $p_{1}(z)=\sigma^{3}(z), p_{2}(z)=$ $\sigma^{3}(z) \wp(z)$ and $p_{3}(z)=\sigma^{3}(z) \wp^{\prime}(z)$. Then

$$
\mathbf{p}: \mathbb{C} \rightarrow E(\mathbb{C})
$$

given by $\mathbf{p}(z)=\left(p_{1}(z), p_{2}(z), p_{3}(z)\right)$ gives a parameterization of the complex points on $E$. We recall that the ring of endomorphisms $\mathscr{O} \subseteq \mathbb{C}$ of $E$ is either $\mathbb{Z}$ or an order in an imaginary quadratic field. If $\omega_{1}$ and $\omega_{2}$ denote generators for the lattice of periods of $\wp(z)$ then in the latter case $\mathscr{O}=\mathbb{Z}+\rho \mathbb{Z}$ where $\rho$ is an algebraic integer in $\mathbb{Q}(\tau), \tau=\omega_{2} / \omega_{1}$. In this case let $\mathscr{O}(S)=\mathbb{Z}(S)+\rho \mathbb{Z}(S)$. 
Theorem 2. Suppose that $E$ is an elliptic curve defined over $\overline{\mathbb{Q}}$ with endomorphism ring $\mathscr{O} \subseteq \mathbb{C}$. Let $\left\{u_{1}, \ldots, u_{m}\right\}$ and $\left\{v_{1}, \ldots, v_{n}\right\}$ be $\mathcal{O}$-linearly independent sets of complex numbers.

(a) Put $\delta=(m+2 n) /(n+1)$. Suppose that there exists $S_{0} \geq 0$ such that for $s_{i} \in \mathscr{O}(S), 1 \leq i \leq m$, and $t_{j} \in \mathscr{O}(S), 1 \leq j \leq n$, with $S \geq S_{0}$ one has

$$
\left|\sum_{i=1}^{m} s_{i} u_{i}\right|>\exp \left(-\min \left(S \log S, S^{m / \delta(3 \delta+2)}\right)\right)
$$

and

$$
\left|\sum_{j=1}^{n} t_{j} v_{j}\right|>\exp \left(-S^{2 m / \delta(\delta-2)}\right) .
$$

If $m>\delta$ then

$$
\text { trans } \operatorname{deg}_{\mathbb{Q}} \mathbb{Q}\left(u_{i}, \wp\left(u_{i} v_{j}\right): 1 \leq i \leq m, 1 \leq j \leq n\right)^{*} \geq \llbracket \frac{m}{\delta} \rrbracket,
$$

where * indicates that we delete $\wp\left(u_{i} v_{j}\right)$ from the generators of this field if it is undefined.

(b) If the hypotheses of part (a) hold with $\delta=(m+2 n) / n$ and $n \geq m>\delta$ then

$$
\text { trans } \operatorname{deg}_{\mathbb{Q}} \mathbb{Q}\left(\wp\left(u_{i} v_{j}\right): 1 \leq i \leq m, 1 \leq j \leq n\right)^{*} \geq \llbracket \frac{m}{\delta} \rrbracket .
$$

(c) Suppose $\mathscr{O} \neq \mathbb{Z}$. If the hypotheses of part (a) hold with $\delta=$ $(2 m+2 n) /(n+1)$ and the right-hand side of (14) (respectively (15)) replaced by

$$
\exp \left(-\min \left(S \log S, S^{2 m / \delta(3 \delta+2)}\right) \quad \text { (respectively } \exp \left(-S^{2 m / \delta(3 \delta-4)}\right)\right)
$$

then $2 m>\delta$ implies that

$$
\text { trans } \operatorname{deg}_{\mathbb{Q}} \mathbb{Q}\left(u_{i}, \wp\left(u_{i} v_{j}\right): 1 \leq i \leq m, 1 \leq j \leq n\right)^{*} \geq \llbracket \frac{2 m}{\delta} \rrbracket .
$$

(d) Suppose $\mathscr{O} \neq Z$. If the lower bounds described in part (c) hold with $\delta=(2 m+2 n) / n$ and $2 m>\delta$ then

$$
\text { trans } \operatorname{deg}_{\mathbb{Q}} \mathbb{Q}\left(\wp\left(u_{i} v_{j}\right): 1 \leq i \leq m, 1 \leq j \leq n\right)^{*} \geq \llbracket \frac{2 m}{\delta} \rrbracket .
$$

Remarks. 1. As a corollary of Theorem 2 we obtain [15, Corollary 3].

2. Theorem 2 implies stronger versions of Corollaire 13.1 (when $n=1$ ) and of Corollaire 13.3 (when $n=1$ ) of [17]. It also improves the main result of [3] and of [4].

Moreover we have the following result concerning the elliptic GelfondSchneider Conjecture which improves the one due to Waldschmidt [17]. 
Corollary. Suppose $\beta$ is algebraic of degree $d \geq 3$ over the field of fractions of $\mathscr{O}$ and let $u$ be a complex number such that $\wp(z)$ is defined at $u, \beta u, \ldots, \beta^{d-1} u$ with $\wp(u)$ algebraic.

Put

$$
t= \begin{cases}\llbracket \frac{d+1}{3} \rrbracket & \text { if } \mathscr{O}=\mathbb{Z} \\ \llbracket \frac{d+1}{2} \rrbracket & \text { if } \mathscr{O} \neq \mathbb{Z} .\end{cases}
$$

At least $t$ of the values

$$
\wp(\beta u), \ldots, \wp\left(\beta^{d-1} u\right)
$$

are algebraically independent.

To deduce Theorem 2 from the main theorem we need a description of algebraic subgroups of $E^{n}$ in terms of the vanishing of linear forms in the tangent space. A crucial point is that we need to have bounds on the coeffcients of these linear forms in terms of the degree of the algebraic subgroup. When $E$ does not have complex multiplication such a result was established by Masser and Wüstholz in [9]; when $E$ has complex multiplication the present author gave such a result in [15], (Lemma 2). However to deduce Theorem 2 with the above explicit lower bounds on the modulus of linear forms in $u_{1}, \ldots, u_{m}$ or $v_{1}, \ldots, v_{n}$ we need the following result.

Proposition 2. Let $E$ be an elliptic curve defined over $\mathbb{C}$. Suppose that $W$ is a subgroup of $\mathbb{C}^{n}$ and that $P_{1}, \ldots, P_{m}$ are multihomogeneous polynomials of degree at most $D$ such that $Z\left(P_{1}, \ldots, P_{m}\right) \cap E^{n}$ is a connected algebraic subgroup of dimension $n-r$. Suppose further that for all $\mathbf{w}=\left(w_{1}, \ldots, w_{n}\right) \in$ $W$,

$$
P_{j}\left(\mathbf{p}\left(w_{1}\right), \ldots, \mathbf{p}\left(w_{n}\right)\right)=0, \quad 1 \leq j \leq m .
$$

Then there exists 1 -linearly independent non-zero vectors

$$
t^{(j)} \in \mathscr{O}_{ \pm}\left(c_{35} D^{r / 2(r+1-j)}\right)
$$

for $j=1, \ldots, r$, such that

$$
t^{(j)} \cdot \mathbf{w} \in \mathscr{L} \text { for all } \mathbf{w} \ni W .
$$

Proof. This is a special case of [2,Theorem 2$]$.

We now establish Theorem 2. To convince the reader that the hypotheses on $u_{1}, \ldots, u_{m}$ and $v_{1}, \ldots, v_{n}$ are necessary we focus on the complex multiplication case and deduce Theorem 2 part (c) from the main theorem.

To establish Theorem 2 we consider the algebraic group $G=\mathbb{G}_{a} \times E^{n}$, with the one-parameter subgroup $\phi(z)=\left(z, \mathbf{p}\left(v_{1} z\right), \ldots, \mathbf{p}\left(v_{n} z\right)\right)$, at the points $y_{i}=u_{i}$ and $y_{i+m}=\tau u_{i}$. Then to deduce this result from our main theorem we show that the hypothesis of Theorem 2 imply those of the main theorem. 
Without loss of generality assume that all of $\wp\left(u_{i} v_{j}\right)$ are defined and put $\boldsymbol{\theta}=\left(g_{2}, g_{3}, y_{1}, \ldots, y_{2 m}, \wp\left(v_{1} y_{1}\right), \wp^{\prime}\left(v_{1} y_{1}\right), \ldots, \wp\left(v_{n} y_{2 m}\right), \wp^{\prime}\left(v_{n} y_{2 m}\right)\right)$.

Recall that $\mathscr{J}$ is the ideal of polynomials over $\mathbb{C}$ which vanish at $\theta$. We show that the hypotheses of Theorem 2 imply that Condition 1 holds for all $\tilde{\theta} \in B(\theta, \exp (-\Delta)) \cap Z(\mathscr{J})$.

If not, there exists an infinite sequence of real numbers $\left\{S_{i}\right\}$ such that for each $i$ there exists $\tilde{\theta}_{i} \in B(\theta, \exp (-\Delta)) \cap Z(\mathscr{J})$ and a connected algebraic subgroup $\widetilde{G}_{i}^{\prime}$ of $\widetilde{G}_{i}$ (which is incompletely defined modulo $\widetilde{G}_{i}$ by polynomials of multidegree at most $\left(L_{0}, L_{1}, D\right)$, where we have not displayed the dependence of each of $\Delta, L_{0}, L_{1}$, and $D$ on $S_{i}$ ) such that

$$
\operatorname{card}\left(\frac{\widetilde{\Gamma}\left(S_{i}\right)+\widetilde{G}_{i}^{\prime}}{\widetilde{G}_{i}^{\prime}}\right)<\frac{H\left(\widetilde{G}_{i} ; L_{0}, L_{1}, 2 D\right)}{H\left(\widetilde{G}_{i}^{\prime} ; L_{0}, L_{1}, D\right)} .
$$

If we take $S_{i}$ to be sufficiently large then $\tilde{g}_{2}=g_{2}$ and $\tilde{g}_{3}=g_{3}$. Therefore $\widetilde{G}_{i}=G=G_{a} \times E^{n}$. Also, in the situation of Theorem 2 we have $L_{1}=0$ and thus (16) reduces to

$$
\operatorname{card}\left(\frac{\widetilde{\Gamma}\left(S_{i}\right)+\widetilde{G}_{i}^{\prime}}{\widetilde{G}_{i}^{\prime}}\right)< \begin{cases}c_{36} L_{0} D^{r} & \text { if } \widetilde{G}_{i}^{\prime} \cong E^{n-r} \\ c_{37} D^{4} & \text { if } \widetilde{G}_{i}^{\prime} \cong \mathbb{G}_{a} \times E^{n-r} .\end{cases}
$$

Recalling that $L_{0}=S_{i}^{\delta}\left(\log S_{i}\right)^{\rho-1}$ and $D=S_{i}^{\delta-2}\left(\log S_{i}\right)^{\rho}$ with $\delta=$ $(2 m+2 n) /(n+1)$ an (easy) calculation shows that if $\widetilde{G}_{i}^{\prime} \cong E^{n-r}$ then

$$
\operatorname{card}\left(\frac{\widetilde{\Gamma}\left(S_{i}\right)+\widetilde{G}_{i}}{\widetilde{G}_{i}^{\prime}}\right)<S_{i}^{2 m},
$$

provided we take $S_{i}$ to be sufficiently large.

Thus there exists $\tilde{\gamma} \in \widetilde{\Gamma}\left(S_{i}\right) \cap \widetilde{G}_{i}^{\prime}$ which is non-trivial. If we recall the points $\widetilde{P}_{i}$ which are obtained from $P_{i}$ above, (3), by replacing $\theta$ by $\tilde{\theta}$ we deduce that there exist $\tilde{y}_{i}, \tilde{w}_{i 1}, \ldots, \tilde{w}_{i n}, 1 \leq i \leq 2 m$, in $\mathbb{C}$ such that

$$
\tilde{P}_{i}=\left(\tilde{y}_{i}, \mathbf{p}\left(\tilde{w}_{i 1}\right), \ldots, \mathbf{p}\left(\tilde{w}_{i n}\right)\right), \quad 1 \leq i \leq 2 m,
$$

projecting onto $\mathbb{G}_{a}$ yields

$$
\sum_{i=1}^{2 m} s_{i} \tilde{y}_{i}=0
$$

Replacing $\tilde{y}_{i}$ by $y_{i}$ we obtain

$$
\left|\sum_{i=1}^{2 m} s_{i} y_{i}\right|<m S \exp \left(-c_{39} \Delta\right)
$$


This last inequality contradicts the measure of linear independence for $u_{1}$, $\ldots, u_{m}$ over $\mathscr{O}$. Hence we must have $\widetilde{G}_{i}^{\prime} \cong \mathbb{G}_{a} \times E^{n-r}$.

We then deduce that

$$
\operatorname{card}\left(\frac{\widetilde{\Gamma}\left(S_{i}\right)+\widetilde{G}_{i}^{\prime}}{\widetilde{G}_{i}^{\prime}}\right)< \begin{cases}S_{i}^{2 m-2} & \text { if } r=1 \\ S_{i}^{2 m} & \text { if } r \geq 2,\end{cases}
$$

provided we take $S_{i}$ to be sufficiently large.

Suppose $r \geq 2$. Then by the above estimates there exists a non-trivial $\tilde{\gamma} \in$ $\widetilde{\Gamma}(S) \cap \widetilde{G}_{i}^{\prime}, \tilde{\gamma}=\mathbf{s} \cdot\left(\widetilde{P}_{1}, \ldots, \widetilde{P}_{2 m}\right)$, with $\widetilde{P}_{i}$ represented above. As $r \geq 2$ there exist $\mathscr{O}$-linearly independent vectors $\mathbf{t}^{(1)} \in \mathscr{O}_{ \pm}^{n}\left(d_{40} D^{1 / 2}\right)$ and $\mathbf{t}^{(2)} \in \mathscr{O}_{ \pm}^{n}\left(c_{41} D\right)$ such that with $\tilde{\boldsymbol{\omega}}_{j}=\left(\tilde{\boldsymbol{\omega}}_{1 j}, \ldots, \tilde{\boldsymbol{\omega}}_{m j}\right)$ we have

$$
\mathbf{t}^{(k)} \cdot\left(\mathbf{s} \cdot \tilde{\boldsymbol{\omega}}_{1}, \ldots, \mathbf{s} \cdot \tilde{\boldsymbol{\omega}}_{n}\right)=\left(m^{(k)}+n^{(k)} \tau\right) \omega_{1}, \quad k=1,2,
$$

where $\tau=\omega_{2} / \omega_{1}$. There exists $a \in \mathbb{Z}$ with $|a|<c_{42}$ such that $a \tau \in \mathscr{O}$ therefore we obtain

$$
\mathbf{t}^{\prime(k)} \cdot\left(\mathbf{s} \cdot \tilde{\boldsymbol{\omega}}_{1}, \ldots, \mathbf{s} \cdot \tilde{\boldsymbol{\omega}}_{n}\right)=\sigma^{(j)} \omega_{1}, \quad k=1,2,
$$

with $\left|\mathbf{t}^{(k)}\right| \leq c_{43}\left|\mathbf{t}^{(k)}\right|$ and $\sigma^{(k)} \mathbf{s} \cdot \tilde{\boldsymbol{\omega}} \in \mathcal{O}$. We then eliminate the common occurrence of $\omega_{1}$ from these two equations to obtain

$$
\mathbf{t} \cdot\left(\mathbf{s} \cdot \tilde{\boldsymbol{\omega}}_{1}, \ldots, \mathbf{s} \cdot \tilde{\boldsymbol{\omega}}_{n}\right)=0
$$

with $\mathrm{t} \in \mathscr{O}_{ \pm}^{n}\left(c_{44} D^{3 / 2} S\right)$. Replacing $\tilde{\omega}_{i j}$ by $y_{i} v_{j}$ for each $i, j$ we obtain

$$
\left|\sum_{j=1}^{n} t_{j} v_{j}\right|\left|\sum_{i=1}^{2 m} s_{i} y_{i}\right|<c_{45} D^{3 / 2} S^{2} \exp \left(-c_{46} \Delta\right) \text {. }
$$

This contradicts our lower bound for the $\mathscr{O}$-linear independence of either $\left\{u_{1}, \ldots, u_{m}\right\}$ or $\left\{v_{1}, \ldots, v_{n}\right\}$.

Therefore we must have $r=1$. But then (18) together with an application of $[8$, Lemma 3$]$ implies that there exist three $\mathbb{Z}$-linearly independent vectors $\mathbf{s}^{(1)}, \mathbf{s}^{(2)}, \mathbf{s}^{(3)} \in \mathbb{Z}_{ \pm}^{2 m}\left(c_{47} S\right)$ such that

$$
\gamma^{(k)}=\mathbf{s}^{(k)} \cdot\left(\widetilde{P}_{1}, \ldots, \widetilde{P}_{2 m}\right) \in \widetilde{G}_{i}^{\prime}, \quad k=1,2,3 .
$$

As $r=1$, Proposition 2 implies that there exists a nonzero vector $\mathbf{t} \epsilon$ $\mathscr{O}_{ \pm}^{n}\left(c_{48} D^{1 / 2}\right)$ such that

$$
\mathbf{t} \cdot\left(\mathbf{s}^{(k)} \tilde{\boldsymbol{\omega}}_{1}, \ldots, \mathbf{s}^{(k)} \tilde{\boldsymbol{\omega}}_{n}\right)=m^{(k)} \omega_{1}+n^{(k)} \omega_{2}, \quad k=1,2,3 .
$$

Hence we perform eliminations over $\mathbb{Z}$ to obtain

$$
\mathbf{t} \cdot\left(\mathbf{s} \cdot \tilde{\boldsymbol{\omega}}_{1}, \ldots, \mathbf{s} \cdot \tilde{\boldsymbol{\omega}}_{n}\right)=0
$$


with $\mathbf{s} \in \mathbb{Z}_{ \pm}^{2 m}\left(c_{49} D^{3 / 2} S^{4}\right)$. Replacing $\tilde{\omega}_{i j}$ by $y_{i} v_{j}$ we obtain

$$
\left|\sum_{j=1}^{n} t_{j} v_{j}\right|\left|\sum_{i=1}^{2 m} s_{i} y_{i}\right|<c_{50} D^{2} S^{4} \exp \left(-c_{15} \Delta\right),
$$

which for $S_{i}$ sufficiently large contradicts our measures of $\mathscr{O}$-linear independence for either $\left\{u_{1}, \ldots, u_{m}\right\}$ or $\left\{v_{1}, \ldots, v_{n}\right\}$.

This establishes Theorem 2 part (c).

Theorems 1 and 2 have abelian analogues wherein one investigates the algebraic independence of coordinates of points given as values of abelian functions. We give here only one such result.

Suppose that $A$ is a principally polarized simple abelian variety of dimension $g$ which is defined over $\overline{\mathbb{Q}}$. Assume that $f_{1}, \ldots, f_{g}$ are the abelian functions associated with $A$, that is, they are meromorphic functions on $\mathbb{C}^{g}$ which are algebraically independent; moreover, if $\mathscr{L} \subseteq \mathbb{C}^{g}$ denotes the period lattice for these functions then $A \cong \mathbb{C}^{g} / \mathscr{L}$. Let $\|\cdot\|$ denote a norm on $\mathbb{C}^{g}$.

Theorem 3. Suppose that $A$ has no complex multiplications, that is, $\operatorname{End}(A) \cong \mathbb{Z}$. Let $u_{1}, \ldots, u_{m}$ be $\mathbb{Z}$-linearly complex numbers and $\mathbf{v}_{1}, \ldots, \mathbf{v}_{n}$ be $\mathbb{Z}$-linearly elements of $\mathbb{C}^{g}$. Let $\delta=(m+2 n g) / n g$. Assume that there exists $S_{0}>0$ such that for all $\mathbf{s} \in \mathbb{Z}^{m}(S)$ and all $\mathbf{t} \in \mathbb{Z}^{n}(S)$, with $S \geq S_{0}$.

$$
\left|\sum_{i=1}^{n} s_{i} u_{i}\right|>\exp \left(-\min \left(S \log S, S^{m / 2 \delta(2 g+\delta)}\right)\right)
$$

and

$$
\left\|\sum_{j=1}^{n} t_{j} v_{j}\right\|>\exp \left(-S^{m / 2 \delta(\delta-1) 8 g^{3} n}\right) .
$$

If $m n>2 m+2 n g$, then at least $\llbracket m / \delta \rrbracket$ of the numbers

$$
f_{k}\left(\mathbf{v}_{j} u_{i}\right) \quad(1 \leq k \leq g, \quad 1 \leq j \leq n, \quad 1 \leq i \leq m)
$$

are defined and are algebraically independent.

This improves an earlier result of Waldschmidt's ([17, Corollaire 15.2]). Our proof is based on the following Proposition due to Bertrand ([2, Theorem 2]).

Proposition 3. Suppose that $A$ is a simple abelian variety of dimension $g$ which is defined over $\overline{\mathbb{Q}}$ and for which $\operatorname{End}(A) \cong \mathbb{Z}$. Suppose that $B$ is 
an abelian subvariety of $A^{n}$ which is incompletely defined modulo the ideal defining $A^{n}$ in multiprojective space by polynomials of multidegree at most $D$. Assume moreover that $B$ is isogeneous to $A^{m}$.

Then there exist $\mathbb{Z}$-linearly independent vectors

$$
\mathbf{t}^{(j)} \in \mathbb{Z}^{g}\left(c_{52} D^{n-m / 2 g(n-m+j-1)}\right), \quad 1 \leq j \leq n-m,
$$

such that for all $\left(\mathbf{y}_{1}, \ldots, \mathbf{y}_{n}\right) \in\left(\mathbb{C}^{\mathcal{g}}\right)^{n}$ with $\exp _{A^{n}}(\mathbf{y}) \in B$,

$$
\sum_{i=1}^{n} t_{i}^{(j)} \mathbf{y}_{i} \in \mathscr{L} .
$$

We now establish Theorem 3.

Proof of Theorem 3. The algebraic group under consideration is $G=$ $A^{n}$; we suppose that $A$ is defined over $\mathbb{Q}(\zeta) \subseteq \overline{\mathbb{Q}}$. There exists a function $h(\mathbf{z})$ algebraically dependent over $\overline{\mathbb{Q}}$ on $f_{1}(\mathbf{z}), \ldots, f_{g}(\mathbf{z})$ such that $\mathbb{C}\left(f_{1}, \ldots, f_{g}, h\right)$ contains all functions which are abelian with respect to the lattice of periods $\mathscr{L}$ of $f_{1}, \ldots, f_{g}$. Moreover, there exists an entire function $\theta(\mathbf{z})$, a theta function with respect to $\mathscr{L}$, such that

$$
\exp _{A}(\mathbf{z})=\left(\theta f_{1}(\mathbf{z}), \ldots, \theta f_{g}(\mathbf{z}), \theta h(\mathbf{z})\right) .
$$

We take as our one-parameter subgroup of $A^{n}$ the function

$$
\phi(z)=\left(\exp _{A}\left(\mathrm{v}_{1} z\right), \ldots, \exp _{A}\left(\mathrm{v}_{n} z\right)\right) .
$$

To simplify our deduction we assume that each of $f_{k}\left(v_{j} u_{i}\right)$ is defined. We then let

$$
\begin{aligned}
\boldsymbol{\theta}=( & \left(\zeta, f_{1}\left(\mathbf{v}_{1} u_{1}\right), \ldots, f_{g}\left(\mathbf{v}_{1} u_{1}\right), h\left(\mathbf{v}_{1} u_{1}\right),\right. \\
& \left.\ldots, f_{1}\left(\mathbf{v}_{n} u_{m}\right), \ldots, f_{g}\left(\mathbf{v}_{n} u_{m}\right), h\left(\mathbf{v}_{m} u_{m}\right)\right) \in \mathbb{C}^{n m(g+1)+1} .
\end{aligned}
$$

Suppose that there exist infinitely many real numbers $S_{i}$ such that for each $i$ there exists $\tilde{\theta}$ with $|\theta-\tilde{\theta}|<\exp \left(-\Delta_{i}\right)$ and a connected algebraic subgroup $\tilde{G}_{i}^{\prime}$ of $\widetilde{G}_{i}$, of codimension $r$ such that

$$
\operatorname{card}\left(\frac{\widetilde{\Gamma}\left(S_{i}\right)+\widetilde{G}_{i}^{\prime}}{\widetilde{G}_{i}^{\prime}}\right)<\frac{H\left(\widetilde{G}_{i} ; 2 D_{i}\right)}{H\left(\widetilde{G}_{i}^{\prime} ; D_{i}\right)} \leq c_{53} D_{i}^{\prime} .
$$

As before, if $S_{i}$ is sufficiently large then $\widetilde{G}_{i}=G$. We consider two cases. If $1 \leq r \leq 2 g$ then the inequality $c_{54} D^{r}<S_{i}^{m-2 g}$ for $m n>$ $2 m-2 n g$, provided $S_{i}$ is sufficiently large, implies that there exist $2 g+1$ vectors $\mathbf{s}^{(k)} \in \mathbb{Z}^{m}(S)$ which are $\mathbb{Z}$-linearly independent vectors such that $\mathbf{s}^{(k)} \cdot\left(\widetilde{P}_{1}, \ldots, \widetilde{P}_{m}\right) \in \widetilde{G}_{i}^{\prime}$. 
We note that this is exactly the point where we need the inequality $m n>$ $2 m+2 n g$ rather than the weaker one $m>\delta$. Choose $\tilde{\boldsymbol{\omega}}_{i j} \in \mathbb{C}^{g}$ with $\left\|\mathrm{v}_{j} u_{i}-\omega_{i j}\right\|<\exp (-\Delta)$ such that

$$
\left(\exp _{A}\left(\tilde{\boldsymbol{\omega}}_{i 1}\right), \ldots, \exp _{A}\left(\tilde{\boldsymbol{\omega}}_{i n}\right)\right)=\widetilde{P}_{i}, \quad 1 \leq i \leq m .
$$

By Proposition 3 above, there exists a non-trivial vector $\mathrm{t}=\left(t_{1}, \ldots, t_{n}\right) \in$ $\mathbb{Z}^{n}\left(c_{55} D^{1 / 2 g}\right)$ such that

$$
t_{1}\left(s_{1}^{(k)} \tilde{\boldsymbol{\omega}}_{11}+\cdots+s_{m}^{(k)} \tilde{\boldsymbol{\omega}}_{m 1}\right)+\cdots+t_{n}\left(s_{1}^{(k)} \tilde{\boldsymbol{\omega}}_{1 n}+\cdots+s_{m}^{(k)} \tilde{\boldsymbol{\omega}}_{m n}\right)=W_{k} \in \mathscr{L} .
$$

Since the $\mathbb{Z}$-rank of $\mathscr{L}$ is $2 g$, there exists a $\mathbb{Z}$-linear combination

$$
\sum_{j=1}^{2 g+1} l_{j} W_{j}=0
$$

and by Siegel's lemma we can take

$$
\max _{1 \leq k \leq 2 g+1}\left|l_{k}\right| \leq c_{56} \max _{1 \leq k \leq 2 g+1}\left|W_{k}\right|^{2 g} .
$$

Yet we have $\max \left|W_{k}\right| \leq c_{57} D^{1 / 2 g} \cdot S$ and therefore

$$
\max \left|l_{k}\right| \leq c_{58} D S^{2 g} \text {. }
$$

Then

$$
t_{1}\left(\sum_{k=1}^{2 g+1} \sum_{i=1}^{m} l_{k} s_{i}^{(k)} \tilde{\boldsymbol{\omega}}_{i 1}\right)+\cdots+t_{n}\left(\sum_{k=1}^{2 g+1} \sum_{i=1}^{m} l_{k} s_{i}^{(k)} \tilde{\boldsymbol{\omega}}_{i n}\right)=0
$$

Replacing $\tilde{\omega}_{i j}$ by $\mathbf{v}_{j} \cdot u_{i}(1 \leq i \leq m, 1 \leq j \leq n)$ and rewriting we deduce that

$$
\left\|\left(\sum_{j=1}^{n} t_{j} \mathbf{v}_{j}\right)\left(\sum_{i=1}^{m}\left(\sum_{k=1}^{2 g+1} l_{k} s_{i}^{(k)}\right) u_{i}\right)\right\|<c_{59} \exp \left(-c_{60} \Delta\right) .
$$

This inequality violates our hypotheses on $\left\{u_{1}, \ldots, u_{m}\right\}$ or $\left\{\mathbf{v}_{1}, \ldots, \mathbf{v}_{n}\right\}$ above.

We now consider the case $r \geq 2 g+1$. Then there exists $\mathbf{s} \in \mathbb{Z}^{m}(S)$ such that $\mathbf{s} \cdot\left(\widetilde{P}_{1}, \ldots, \widetilde{P}_{m}\right) \in \widetilde{G}_{i}^{\prime}$. Also, by Proposition 3 above there exist vectors

$$
\mathbf{t}^{(k)} \in \mathbb{Z}^{n}\left(c_{61} D^{r / 2 g(r+k-1)}\right), \quad 1 \leq k \leq 2 g+1,
$$

such that if we choose $\tilde{\boldsymbol{\omega}}_{i j}$ as above then

$$
t_{1}^{(k)}\left(s_{1} \tilde{\boldsymbol{\omega}}_{11}+\cdots+s_{m} \tilde{\boldsymbol{\omega}}_{m 1}\right)+\cdots+t_{n}^{(k)}\left(s_{1} \tilde{\boldsymbol{\omega}}_{1 n}+\cdots+s_{m} \tilde{\boldsymbol{\omega}}_{m n}\right)=W_{k} \in \mathscr{L}
$$

for $1 \leq k \leq 2 g+1$. Again we apply Siegel's lemma to find $l_{k} \in \mathbb{Z}$ with

$$
\max _{1 \leq k \leq 2 g+1}\left|l_{k}\right| \leq c_{62} D^{r / r+2 g} S^{2 g}
$$


such that $\sum_{k=1}^{2 g+1} l_{k} W_{k}=0$. Therefore

$$
\sum_{k=1}^{2 g+1} l_{k}\left(\sum_{j=1}^{n} t_{j}^{(k)}\left(\sum_{i=1}^{m} s_{i} \tilde{\boldsymbol{\omega}}_{i j}\right)\right)=0 .
$$

Replacing $\tilde{\boldsymbol{\omega}}_{i j}$ by $\mathbf{v}_{j} u_{i}$ we deduce that

$$
\left\|\left(\sum_{j=1}^{n} \sum_{k=1}^{2 g+1} l_{k} t_{j}^{(k)} \cdot \mathbf{v}_{j}\right)\left(\sum_{i=1}^{m} s_{i} u_{i}\right)\right\|<c_{63} \exp \left(-c_{64} \Delta\right),
$$

which contradicts the hypotheses of the Theorem. Thus Condition 1 holds and Theorem 3 may be deduced from the Main Theorem.

Finally we remark that other applications are possible and in particular we obtain an improvement of [17, Corollaire 15.2].

\section{Acknowledgements}

Thanks are extended to Patrice Philippon who made some valuable comments on a preliminary version of this manuscript and pointed out that $\mathbf{M}$. Ably [1] has recently obtained even stronger results. Moreover, we would like to thank the mathematics department at Macquarie University for its hospitality during the revision of this paper, especially John Loxton for his invitation to come to Australia, Gerry Myerson for his camaraderie and Alf van der Poorten for being distracted enough to allow me to focus on this project.

\section{References}

[1] M. Ably, Independance algebrique pour les groupes algebriques commutafs, (Thèse, Lille, 1989), p. 75.

[2] D. Bertrand, 'Minimal heights and polarizations on abelian varieties', Math. Sciences Research Institute, Berkeley, California (June 1987), p. 18.

[3] W. D. Brownawell, 'Large transcendence degree revisited I. Exponential and non-CM cases', Diophantine Approximation and Transcendence Theory, Seminar Bonn edited by G. Wüstholz, pp. 149-173 (Springer Lecture Notes 1290, 1985).

[4] W. D. Brownawell, R. Tubbs, 'Large transcendence degree revisited II. The CM case', Diophantine Approximation and Transcendence Theory, Seminar Bonn edited by G. Wüstholz, pp. 175-188 (Springer Lecture Notes 1290, 1985).

[5] P. L. Cisjouw and R. Tijdemann, "An auxiliary result in the theory of transcendence numbers', Duke Math. J. 42 (2) (1975), 239-247. 
[6] G. Diaz, 'Grand degrés de transcendance pour des familles d'exponentielles', J. Number Theory 31 (1) (January 1989), 1-23.

[7] J. Fresnel, 'Déformation d'un groupe algébrique', Appendice in 'Groupes algébriques et grands degrés de transcendance', M. Waldschmidt, Acta Math. 156 (1986), 295-302.

[8] D. W. Masser, 'On polynomials and exponential polynomials in several complex variables', Invent. Math. 63 (1981), 81-95.

[9] D. W. Masser, G. Wüstholz, 'Fields of large transcendence degree generated by values of elliptic functions', Invent. Math. 72 (1983), 407-464.

[10] P. Philippon, 'Criteres pour l'independance algébrique', Inst. Hautes Études Sci. Publ. Math. 64.

[11] P. Philippon, 'Un lemme de zéros dans les groupes algébriques commutatifs', Bull. Soc. Math. France 114 (1986), 355-383.

[12] E. Reyssat, 'Approximation algébrique de nombres liés aux fonctions elliptiques et exponentielles', Bull. Soc. Math. France 108 (1980), 47-79.

[13] J. P. Serre, 'Quelques propriétés des groupes algébriques commutatifs'. Appendice II, Nombres Transcendants et Groupes Algébriques, edited by M. Waldschmidt (Astérisque, No. 69-70).

[14] R. Tubbs, 'Algebraic groups and small transcendence degree I', J. Number Theory 25 (3) (1987), 279-307.

[15] R. Tubbs, 'The algebraic independence of three numbers: Schneider's method', Proceedings of the Quebec Conference, edited by J. M. DeKoninck and C. Levesque, pp. 942-967 (Walter de Gruyter).

[16] R. Tubbs, 'A diophantine problem on elliptic curves', Trans. Amer. Math. Soc. 309 (1) (1988), 325-338.

[17] M. Waldschmidt, 'Groupes algébreiques et grands degrés de transcendance', Acta Math. 156 (1986), 253-302.

\section{University of Colorado}

Boulder, Colorado 80309-0426

U.S.A. 